\title{
DEVELOPMENT FOR BREEDING PERFORMANCE MANAGEMENT SYSTEM ON PIG FARMS
}

\author{
Benhai Xiong * , Qingyao Luo, Jianqiang Lu, Liang Yang \\ Institute of Animal Science, Chinese Academy of Agricultural Sciences, State Key Laboratory \\ of Animal Nutrition, Beijing 100094, China \\ * Corresponding author, Institute of Animal Science, Chinese Academy of Agricultural \\ Sciences. No. 2 Yuanmingyuan West Road, Beijing 100094, China, Tel: +86-10-62811680, \\ Fax:+86-10-62815988, Email: Bhxiong@iascaas.net.cn orXiongbenh@263.net.
}

Abstract: The study was conducted to supply systemic and dynamic analysis data to support a better operation on a breeding pig farm with process management, especially in reproduction parameters. A full simulation model on a breeding pig farm running was proposed in the study, and a series of definitions of process parameters related to service performance, farrowing performance and weaning performance was put forward. Some of them are described on the calculating models. The relationship structural database was designed and a set of digital management information system was developed, based on proposed definitions and models by using Visual Basic 6.0. Access databases and Crystal report combined with genetic characteristic of different pig breeds. The System supplies a series of convenient, intelligent input interfaces of original datum, and all different reproduction data can be counted, analyzed and graphically shown, based on different performances in a specific duration, and it can dynamically derive out all sows history card that shows a complete reproduction performances including some important indexes such as farrowing rate, farrowing interval, average gestation days and average weaned weight et al. in terms of parities, which can be used to decide whether a female needs to be fell into disuse. Therefore, with the help of system analysis and software design techniques, the system made it possible to realize information management and intelligence analysis for a breeding pig farm based on whole digital management of reproduction process from services through weaning and among different categories of breeding pigs and parities.

Keywords: Pig farms, Breeding sows, Information management, Farrowing performance

Xiong, B., Luo, Q., Lu, J. and Yang, L., 2008, in IFIP International Federation for Information Processing, Volume 258; Computer and Computing Technologies in Agriculture, Vol. 1; Daoliang Li; (Boston: Springer), pp. 267-275. 


\section{INTRODUCTION}

With the help of advanced science and management technologies, pig farming has gained fast advances, especially in production rate of pigs and utilization efficiency of nutrients around the world. Currently, the most efficient method to reduce cost of pig farming is to enhance reproduction performance of sows by strengthening the whole breeding process. (Rothschild MF, 1996). Besides reproduction techniques themselves, application of information technologies is becoming more and more important due to timely data gathering and just-in-time decision making. Therefore, many information management system have been developed and applied in pig farms, especially those farms with breeding sow (www.pigwin.com; www.agritecsoft.com/en/porcitec/; http://psru.marc.usda.gov). Among these systems, the most core task focus on female process management from mating, gestation, farrowing to weaning. The objective of managements is not only to calculate reproduction performances on individual pigs, but also to know the whole performance of a specific herd so that the producers can find out existing problems.

Although there are different information systems on pig farms abroad, the most important is that we should have own system with our intellectual property rights, and that the differences among language and culture as well as management patterns bring a requirement for designing similar system by using Chinese language. The study consideration based on breeding pig farming will be introduced and a short description was given: (1) a general model of lifecycle for pigs to express a production system of pigs, (2) identification technology adopted for individual pigs, (3) the required recording for reproduction process documents, (4) reproduction performance analysis for females.

\section{FORMULATION OF PROBLEM}

Before developing the management system on breeding pig farms, it is necessary to know what key figures for a breeding pig farm is. With the help of analyzing the key figures, deviations of different female individuals can be found out quickly. If reproduction problems in a farm can timely be recognized, losses will be prevented or reduced to a minimum; especially it can supply basic decision making for culling and updating of basic sows herd. 


\subsection{The categories and production system model on a farm}

Six categories of pigs are distinguished in the reproduction management system, that are suckling piglets, weaned piglets, gilts, empty and pregnant sows, lactating sows and fattening pigs. The key figures can be calculated separately for each of these categories. Fig. 1 shows a whole production system or lifecycle model for pigs and the above categories are located in different processes. Based on the model, their production data can be recorded and integrated in terms of the categories of pigs respectively.

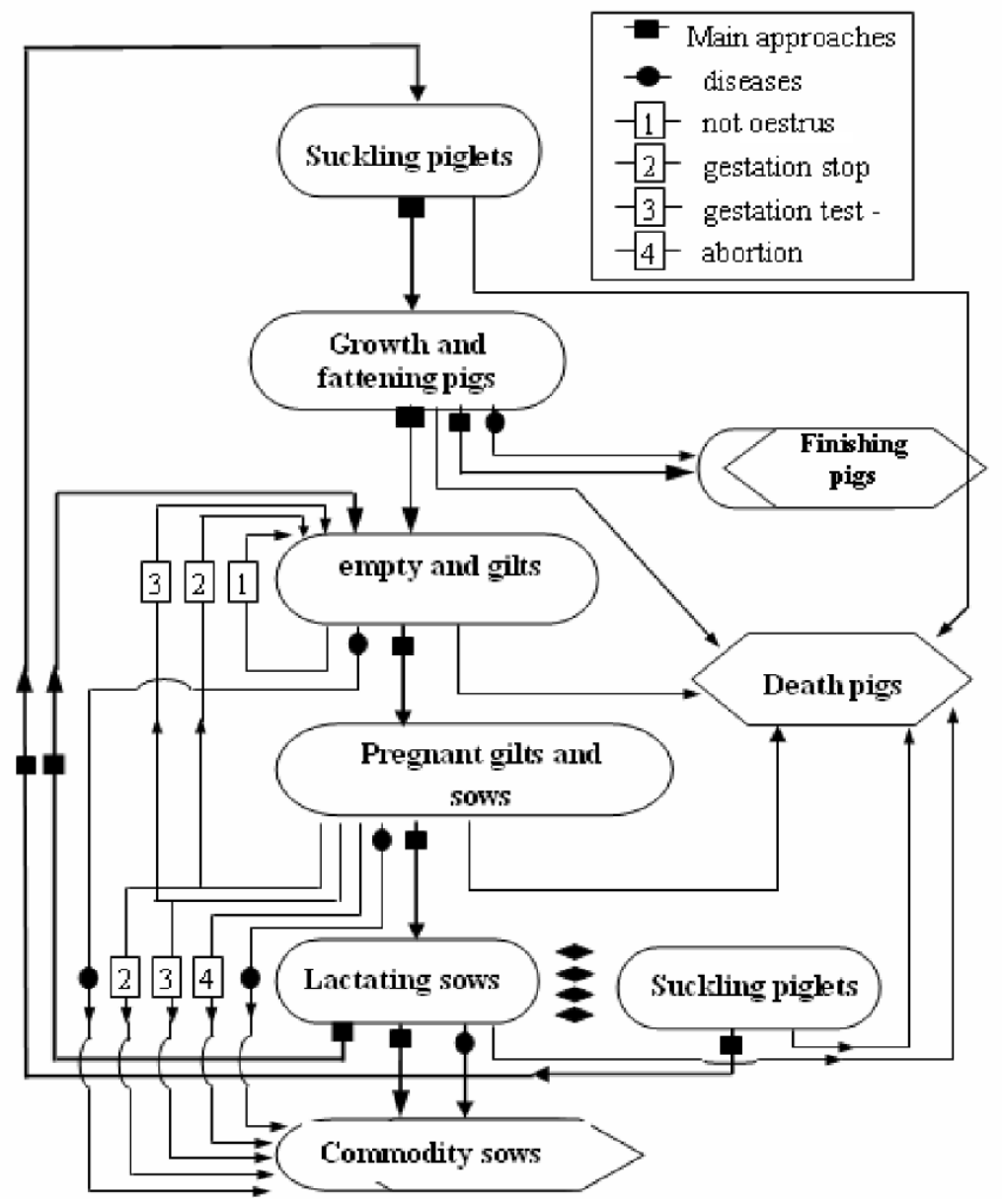

Fig. 1. Production system model sketch map for pigs 


\subsection{Definition of main data items and required recordings}

As known, there are many data items involved in a management information system for a breeding pig farm. Considering length limitation of this paper, the following comprehensive performance analysis indexes for reproduction traits on pigs are shown only. The whole reproduction traits are divided into three parts: service performance, farrowing performance and weaning performance and all indexes are defined in the parts is in the context of one specific period. In the following description, those italic items represent parameters required and to be recorded.

\subsubsection{Service performance}

Total services expresses the total number of services in the period, including repeat services. Number 1st services expresses Services minus Repeat Services. Number repeat services expresses the number of repeat services in the period. Percent repeat services is RepeatServices expressed as a percentage of Services. Number multiple matings expresses the number of services in the period with two or more matings per service. Percent multiple matings is MultipleMatings expressed as a percentage of Services. Matings per service expresses ServMatings divided by Services. Served 1st service after entry expresses the number of entered Unmated Parity 0 females with a first mating event in the period i.e. P0FstServ. Entry to 1st service interval expresses EntryFstServiceDays divided by P0FstServ, where, EntryFstServiceDays represents the sum days from entry to first service for unmated females with their first mating event in the period. Served 1st service after weaning expresses Count of days from the weaning event to the first mating event for females with a first mating post weaning in the period i.e. CountWeanFstServ. Weaning-1st service interval expresses WeanFstServ divided by CountWeanFstServ, where, WeanFstServ represents the sum of days from the wean event to the first mating event for females with a first mating post weaning in the period.

With the help of above 11 items, it is possible to reflect service performance for a female.

\subsubsection{Farrowing performance}

Similarly, the following 13 indexes were adopted in the study to figure out female farrowing performance. Among these items, Farrowing rate, Average gestation and farrowing interval are main parameters that reflect female genetic traits as well as the farm management level. 
Farrowings expresses the number of birthing events in the period i.e. births. Average parity farrowed expresses total birth parity divided by farrowings in the period. Total born per farrow expresses Born divided by Birthings, where, Born means the sum of born alive to all birthing events in the period, Birthings means the number of birthing events in the period. Liveborn per farrow expresses Liveborn divided by Birthings. Stillborn per farrow expresses Stillborn divided by Birthings. Stillborn percent is Stillborn expressed as a percentage of Born. Mummies per farrow expresses Mummies divided by Birthings. Mummies percent is Mummies expressed as a percentage of Born. Farrowing rate is Birthings expressed as a percentage of ServicesPeriodBirth, where ServicesPeriodBirth means the number of females served to farrow in the period.

Average gestation length is SumGestationLen divided by CountGestationLen, where SumGestationLen means the sum days of gestation lengths of females with a birthing event in the period, CountGestationLen means the count of gestation lengths in the period, used for statistical purposes. Farrowing interval expresses days between two consecutive farrowing dates for a mated breeding female. Litters/mated female/year is calculated as GestationDays multiplied by 365 and divided by both 115 and MatedFemaleDays, where GestationDays means the sum of days of all females gestating in the period. A female contributes one gestation day for each day that is between conception and birthing in the period. MatedFemaleDays means the sum of all mated female days. A breeding female is considered as a part of the mated breeding female inventory, effective of its first service date. Lastly, total born/mated female/ year equals to Born multiplied by 365 and divided by MatedFemaleDays.

\subsubsection{Weaning performance}

Compared with farrowing, weaning performance analysis actually reflects the situation of a farm management level. In general, the better a farm management does, the earlier a herd weaning is. These parameters are as follows:

Total pigs weaned expresses the sum of pigs weaned from sows with weaning events in the period, including weaning events of nurse litters. Pigs weaned with a partial weaning event are counted on the date of the following weaning event. Litters Weaned expresses the number of litters weaned in the period, including litters weaned from a nurse on event. Pigs weaned per litter are Total Weaned divided by LittersWeaned. Females weaned or nursed off expresses the number of females with a last wean or nurse off event in the period. Pigs weaned per female expresses LastWeaned divided by FemalesWeaned, where, LastWeaned means total number of animals 
weaned from sows with their last weaning or nurse off event in the period. Net fostered expresses pigs fostered on with a foster event minus pigs fostered off with a foster event plus pigs nursed on with a nurse on event minus pigs nursed off with a nurse off event in the period.

Avg. weaning age is SumWeanLitterAges divided by CountWeanLitterAges, where SumWeanLitterAges means the sum of age at weaning for natural litters weaned in the period, and CountWeanLitterAges means Count of wean litters with weaning ages. Avg weight per weaned pig is SumWeanWeight divided by CountWeanWeight, where SumWeanWeight means the sum of litter weights at weaning for litters weaned in the parity, includes nurse litters, CountWeanWeight means Number of animals weighed at weaning.

Recorded preweaned deaths expresses the sum of preweaned deaths recorded with the PwDeath event in the period. Preweaning mortality rate means pigs that die between live birth and weaning expressed as a percentage of those at risk.

Weaned/mated female/year is TotalWeaned multiplied by 365 and divided by MatedFemaleDays, where TotalWeaned is the sum of pigs weaned from sows with Weaning events in the period, includes Weaning events of nurse litters. Pigs weaned with a Partial Weaning event are counted on the date of the following Weaning event.

Weaned/female/year is TotalWeaned multiplied by 365 and divided by FemaleDays, where femaleDays is the sum of all active female days during the period. A female contributes one female day for each day she was active during the period, beginning at her entry date into the breeding herd and ending with her removal.

Based on the above definition and analysis, as well as computer program design, it is possible to develop a digital and intelligent analyzing module on female breeding performance.

\section{PROBLEM SOLUTION}

\subsection{System program development}

It is divided into two kinds of situations to design this management information system, non-network system and network system. For the first case, access database and VB language were adopted to develop this system and an integrated version is finally supplied for users. For another case, windows server 2003, SQL server 2005 and Visual C\#. Net language were depended upon and it is finally issued it by intranet or internet. 
Before running a pig farm management system, some basic female production theory parameters must be supplied in advance as the references of analysis. The input interface including required setting for growth and reproduction data, and an operator editing these parameters from either theory or management objectives are shown in Fig. 2.

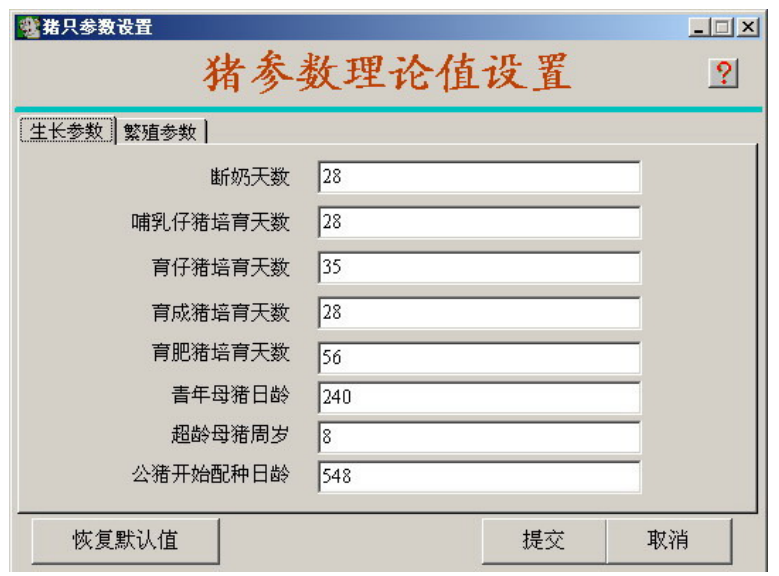

Fig. 2. Setting for theory parameters of a kind of pig

\subsection{Identification to pig individuals}

The identification of individual pigs depends on a national animal labeling program, which has been implementing by Ministry of Agriculture, China. According to this program, every animal (pig, cattle and sheep/goat) has a unique labeling code (http://www.agri.gov.cn/blgg/t20060628_638621.htm, 2006). The labeling code is composed of 15 numeric digits. The first digit expresses livestock category, i.e. 1 swine; 2 cattle, 3 sheep/goats. The following 6 digits $\left(2^{\text {nd }}\right.$ to $\left.7^{\text {th }}\right)$ express the administrative region code that obeys to GB T2260-1999, a national standards of People's Republic of China (http://www.stats.gov.cn/tjbz/xzqhdm/t20070411_402397928.htm,2006), which represents a nature county and is updated on December 31, 2006. The last 8 digits $\left(8^{\text {th }}\right.$ to $\left.15^{\text {th }}\right)$ express a ranking number for a livestock in a specific county in terms of different species respectively. In this system, information or data for individuals is stored in terms of its labeling code.

\subsection{Main output results (example)}

Based on the female performance analysis including service, farrowing and weaning performance, one of the important statistic outputs is female history card. From a specific female history card (Fig. 3), a whole breeding result with a specific female can be found out from parity 0 to current parity 
related to service, farrowing and weaning data. In terms of dynamic changes of performance parameters with a female in different parities, an operator can decide when it shall be fallen into disuse.

Using pre-designed statistic module, the system can analyze the whole female herd and print out results calculated at any time. Formulation of different reports is from both crystal-reporting technology and own designed module.

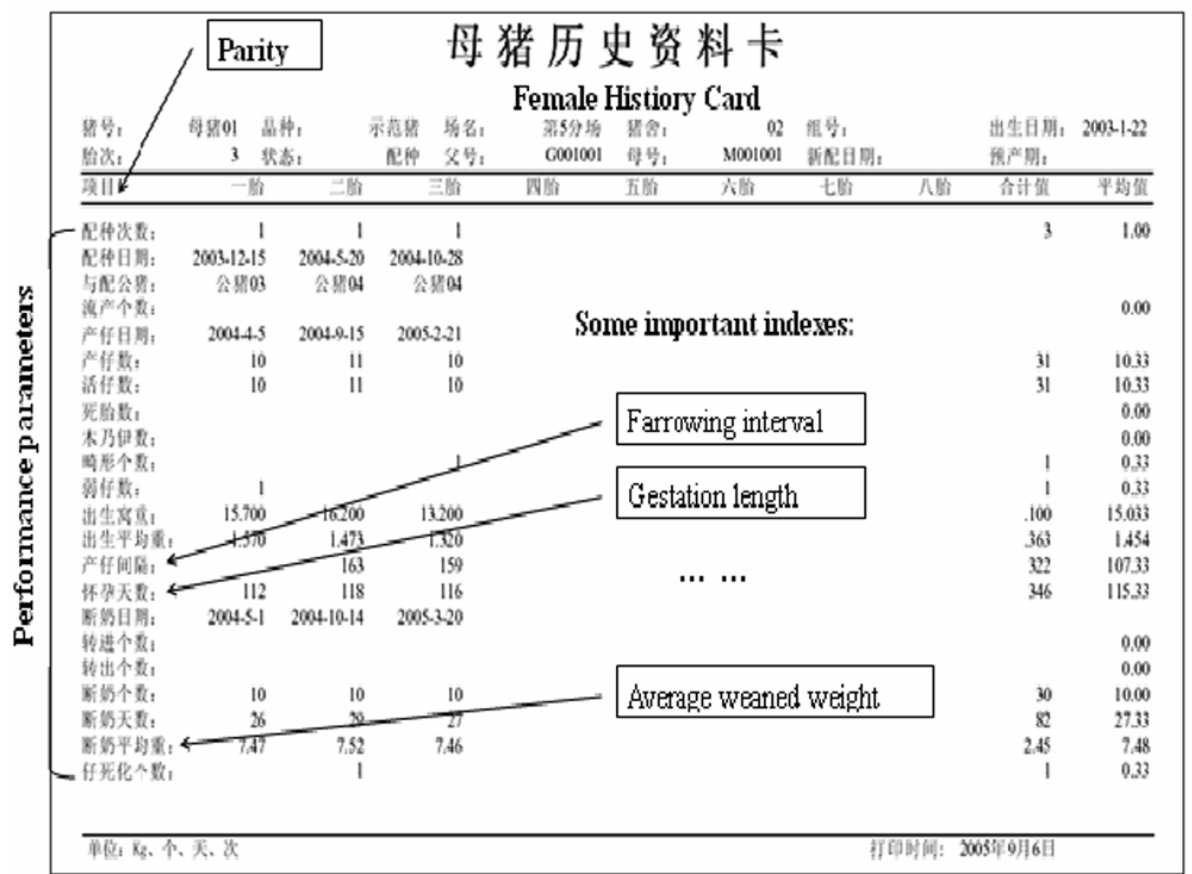

Fig. 3. A female history card (example)

\section{CONCLUSIONS}

The need for a fully operational and economically visible pig farm production management system that is capable to record all process data is highlighted. In order to explore an integrated data gathering and analyzing as well as output of calculated results, a lot of definitions on those data items related to female production performance is presented in the paper. Among these described performance indexes, although most of them are used for individuals of swine, it is also possible to do some herd traits analysis. A practical system focusing on analysis of individual female reproduction process data is proposed also.

Although such a system has realized prime reproduction information management, there are a few aspects to be perfected in the future. Firstly, 
regarding to the method to record process data, an intelligent and mobile PDA embedded system is been developing to record and edit data just in time and upload those gathered data into table-server database system by wireless or GPRS/CDMD (Yao W., 2003; Liu yanbo, 2006) based on network management system that is intranet or internet. The following is the precision feeding for females including gestation sows and lactating sows. The nutrition situation of sows affects up growth of fetuses and growth of piglets. A perfect method is to construct dynamic predicting models to calculate nutrient requirements for individual females in terms of its genetic traits, body conditions and environment factors. All above analysis shows that a good information management system on breeding pig farms needs a lots of professional models on reproduction and nutrition (Xiong B H, 2005).

\section{ACKNOWLEDGEMENTS}

During this paper's writing, Professor Qian Ping from Institute of Agricultural Information of Chinese Academy of Agricultural Sciences gave careful reading of the manuscript and his fruitful comments and suggestions. The author thanks him for his distinguish work.

\section{REFERENCES}

http://psru.marc.usda.gov/

http://www.agri.gov.cn/blgg/t20060628_638621.htm. 2006. No.67th, order of the ministry of agriculture of the people's republic of china. Animal labeling and their feeding documents establishment.

http://www.stats.gov.cn/tjbz/xzqhdm/t20070411_402397928.htm. November, 31, 2006. National

Bureau of Statistics of China. Codes of administrative regions of the People's Republic of China.

Liu yanbo, Hu yan, Ma qi. Platform Application and Development on Windows Mobile. Posts \& Telocom Press. 2006.

Rothschild MF. Genetics and reproduction in the pig. Animal Reproduction Science, 1996 42:143-151.

www.agritecsoft.com/en/porcitec/ www.pigwin.com

Xiong B H, Qian P. A comprehensive technology platform for precision feeding of dairy cattle

[M]. China Agricultural Science and Technology Press. 2005

Yao W. Visual Basic database Development and project examples [M]. Posts and Telecom

Press. 2003. 